\title{
The Mid-IR Spectra of 9-Ethyl Guanine, Guanosine, and 2-Deoxyguanosine ${ }^{\dagger}$
}

\author{
Ali Abo-riziq, ${ }^{\ddagger}$ Bridgit O. Crews, ${ }^{\ddagger}$ Isabelle Compagnon, ${ }^{\S}$ Jos Oomens, ${ }^{\S}$ Gerard Meijer, ${ }^{£}$ \\ Gert Von Helden, ${ }^{\mathfrak{E}}$ Martin Kabeláč," Pavel Hobza," and Mattanjah S. de Vries*,: \\ Department of Chemistry and Biochemistry, University of California Santa Barbara, California 93106-9510, \\ FOM-Institute for Plasmaphysics Rijnhuizen, Edisonbaan 14, NL-3439 MN Nieuwegein, The Netherlands, \\ Fritz-Haber-Institut der Max-Planck-Gesellschaft, Faradayweg 4-6, D-14195 Berlin, Germany, and Institute of \\ Organic Chemistry and Biochemistry and Center for Complex Molecular Systems and Biomolecules, Academy \\ of Sciences of the Czech Republic, 16610 Prague 6, Czech Republic
}

Received: March 19, 2007; In Final Form: May 30, 2007

\begin{abstract}
We present the mid-IR (400-1800 $\mathrm{cm}^{-1}$ ) spectra of 9-ethyl guanine, guanosine, and 2-deoxyguanosine measured by IR-UV double-resonance spectroscopy. We compare the recorded mid-IR spectra with the spectra of the most stable structures obtained from RI-MP2 and RI-DFT-D calculations. The results confirm the enol form for all structures and demonstrate the efficacy of a new approach to DFT calculations that includes dispersion interactions.
\end{abstract}

\section{Introduction}

Since our first report of the sharp resonant two-photon ionization (R2PI) spectrum of guanine ${ }^{1}$ and guanosine ${ }^{2}$ their isomeric structures have commanded considerable scrutiny. In the gas phase under jet-cooled conditions, four tautomers of guanine appear based on hole burning spectroscopy. ${ }^{3-7}$ Remarkably, it now appears that none of these are in the keto form, which is the biologically most relevant type of tautomer because it is the dominant form in solution and it is the form in which Watson-Crick base pairing occurs in DNA. ${ }^{7}$ The identification is based on IR-UV hole burning spectroscopy in the near-IR range. The $\mathrm{OH}$ stretch frequencies serve to clearly identify two of the tautomers as being enol, while the other two show no $\mathrm{OH}$ stretch at all. However, recent analysis has shown these other two tautomers to be the higher energy oxo-imino tautomers rather than the more stable keto tautomers. Choi and Miller have observed the keto tautomers in helium droplets, showing their IR frequencies to differ from those in the R2PI experiments. ${ }^{8}$ Kleinermanns and co-workers have confirmed the oxo-imino assignments for the R2PI case based on the imino $\mathrm{C}-\mathrm{N}$ vibrations at about $1680 \mathrm{~cm}^{-1}$. 9 The difference between the R2PI and the helium droplet work is not only in the degree of cooling but also in the detection method. ${ }^{10}$ All of the R2PI experiments were performed with nanosecond laser pulses, and therefore, they are blind to species with sub-picosecond lifetime excited states. The detection in helium droplets relies on the IR absorption only and does not depend on the excited-state characteristics. It is therefore conceivable that the keto tautomers, which are observed exclusively in the droplets, have significantly shorter excited-state lifetimes than the other forms. A number of models suggest that the excited-state lifetimes in DNA bases are shortened by internal conversion to the ground state, via an intermediate state and mediated by conical intersections. ${ }^{11-18}$

\footnotetext{
† Part of the "Roger E. Miller Memorial Issue".

* Corresponding author. E-mail: devries@chem.ucsb.edu.

$\doteqdot$ University of California Santa Barbara.

$\S$ FOM-Institute for Plasmaphysics Rijnhuizen.

${ }^{£}$ Fritz-Haber-Institut der Max-Planck-Gesellschaft.

\# Academy of Sciences of the Czech Republic.
}

It has been proposed that this type of photochemistry is nature's defense mechanism against photochemical damage.

To further focus on the biologically most relevant form, we have studied guanine with substituents in the $\mathrm{C} 9$ position to mimic the nucleosides, as well as several of the nucleosides themselves. ${ }^{19}$ For all of these species, we observed only a single isomer, which we determined by near IR-UV hole burning to be of the enol form. We now explore these compounds further by reporting IR-UV hole burning in the mid-IR range of 400$1800 \mathrm{~cm}^{-1}$, covering the $\mathrm{C}=\mathrm{O}$ and the $\mathrm{C}-\mathrm{N}$ stretch frequencies to confirm absence of both the keto and imino forms and to further explore the fingerprint frequencies for the sugar moiety. We compare both MP2 and DFT-D computations with the experimental data.

\section{Experimental Method}

We performed the experiment in a previously described laser desorption jet-cooling setup. ${ }^{20-22} \mathrm{We}$ desorbed a mixture of pure compound and some graphite powder from a graphite substrate with pulses from a YAG laser at $1064 \mathrm{~nm}$ and less than $1 \mathrm{~mJ} /$ pulse. We entrained the desorbed molecules in a pulsed supersonic jet of Ar drive gas. We performed mass-selected spectroscopy by resonant two-photon ionization (R2PI), detecting the ions in a linear time-of-flight mass spectrometer. We obtained the IR spectrum by IR-UV hole burning. A few microseconds before the excitation laser was fired, the IR freeelectron laser beam interacted with the molecules in the molecular beam. If a vibrational transition was induced by the IR light, molecular population was transferred from the ground state into an excited vibrational state, resulting in a depletion of the ground-state population. This depletion resulted in a reduction in the number of ions produced by the UV two-photon ionization. By measuring the ion yield of R2PI ions while varying the wavelength of the IR laser, we obtained an ion-dip spectrum.

The IR radiation was produced at the Free Electron Laser for Infrared eXperiments (FELIX) user facility at the FOMInstitute for Plasma Physics in Rijnhuizen, The Netherlands. ${ }^{23}$ The temporal output of this $10 \mathrm{~Hz}$, pulsed laser system consists 
of a $6 \mu$ s long burst (macropulse) of micropulses. The micropulse spacing within the burst was set to $1 \mathrm{~ns}$. The spectral bandwidth was adjusted to approximately $0.5 \%$ (fwhm) of the central frequency, which corresponds to a micropulse duration of about 100 optical cycles. The frequency range that can be covered extends from 40 to $2000 \mathrm{~cm}^{-1}$, although only the range from $500-2000 \mathrm{~cm}^{-1}$ was used in the present study. Typically, energies of up to $100 \mathrm{~mJ}$ can be reached in the macropulse. In the present experiment, the UV detection laser was running at a $10 \mathrm{~Hz}$ repetition rate, while FELIX was set to $5 \mathrm{~Hz}$. By independently recording the alternating IR-on and IRoff signals, we obtained a normalized ion-dip spectrum that is insensitive to long-term drifts in UV laser power or source conditions.

The high intensity of the IR beam can potentially lead to multiphoton absorption. To ensure that our measurements are based on a single-photon absorption, we performed the experiment at a number of IR output powers, converted the normalized signal $s(v)$ into a relative IR absorption cross section $\sigma(v)$ using $\sigma(v)=-\log (s(v))$, and corrected for the IR output power in the scanned region.

\section{Theoretical Method}

We fully optimized the structures of all systems studied by two techniques. (a) We employed the resolution of identity (RI-) MP2 level of theory using the cc-pVDZ basis set with a standard auxiliary basis set. (b) We have recently demonstrated the suitability of DFT theory for the study of biopolymers, when employed in combination with an empirically treated dispersion term (RI-DFT-D). ${ }^{24,25}$ For this purpose, we employed the TPSS functional. ${ }^{26}$ We obtained relative energies and harmonic frequencies at both levels of theory. The numerically calculated harmonic frequencies were scaled by a factor of 0.956 for the RI-MP2 calculations, as used in our previous papers. RI-DFTD-calculated frequencies were not scaled. We used the TURBOMOLE 5.8 program package ${ }^{27}$ with implementation of our code for treating the dispersion term.

\section{Results and Discussion}

Figure 1 shows the tautomeric forms for which we performed the calculations, with the numbering scheme and the nomenclature used in this paper. Figure 2 shows a few of the lowest energy optimized structures. The $\mathrm{n} 3$ forms do not occur in the hydrogen-bonded guanosines since those structures only occur for tautomers with no hydrogen in the N3 position.

Table 1 lists relative stabilities in kilocalories per mole, calculated at the RI-MP2 level, including zero-point vibrational energy. The trends and orders of magnitude are reproduced equally by RI-MP2 and RI-DFT-D methods. The keto and enol structures are always lowest in energy, with a difference of less than $1.5 \mathrm{kcal} / \mathrm{mol}$. The next highest energy structures are the $\mathrm{n} 3$ structures $(1 \mathrm{H}, 3 \mathrm{H}$ imino-oxo) by $12-17 \mathrm{kcal} / \mathrm{mol}$. All other structures are considerably higher in energy. The cis form of the guanosines (stabilized by a hydrogen bond between the N3 on the guanine and the $\mathrm{C}^{\prime}-\mathrm{OH}$ on the sugar) is consistently lower in energy than the extended trans form by $6-8 \mathrm{kcal} / \mathrm{mol}$. Table 2 lists total electronic energies of the keto tautomers calculated at the RI-DFT-D and RI-MP2 levels.

Figure 3 shows the R2PI spectra of 9-ethyl guanine (a), guanosine (b), and 2-deoxyguanosine (c) scanned in the region of $34400-35400 \mathrm{~cm}^{-1}$. According to the UV-UV doubleresonance spectroscopy, these spectra reflect absorption by only a single isomer. On the basis of our IR-UV double-resonance spectroscopy in the region of $3200-3800 \mathrm{~cm}^{-1}$, we assigned<smiles>[R]n1cnc2c(=O)n(C)c(N(C)C)nc21</smiles><smiles>[R]n1cnc2c(OC)nc(N(C)C)nc21</smiles><smiles>[R]n1c(C)nc2c(=O)n(C)c(=NC)n(C)c21</smiles><smiles>[R]n1c(C)nc2c(=O)n(C)c(=NC)n(C)c21</smiles><smiles></smiles><smiles>[R]N1c2nc(NC)n(C)c(=O)c2N(C)C1C</smiles><smiles>[R]n1c(C)nc2c(OC)n(C)c(=NC)nc21</smiles><smiles>[R]n1c(C)nc2c(OC)n(C)c(=NC)nc21</smiles><smiles>[R]n1c(C)nc2c(OC)n(C)c(=NC)nc21</smiles><smiles>[R]n1c(C)nc2c(OC)n(C)c(=NC)nc21</smiles>

Figure 1. Tautomeric forms of 9-substituted guanines.

the structure of this isomer in each of the three compounds as the enol form. ${ }^{19}$ This assignment is consistent with the R2PI spectroscopy of the guanine nucleobase, for which the $9 \mathrm{H}$ enol tautomer has its origin at $34755 \mathrm{~cm}^{-1}$, as compared to the guanosine origin at $34443 \mathrm{~cm}^{-1} \cdot 2,7$ The two guanine tautomers that have no free $\mathrm{OH}$ stretch frequency in the IR-UV spectra have their origins at 32864 and $33269 \mathrm{~cm}^{-1}$, respectively. ${ }^{4} \mathrm{We}$ scanned down to $30000 \mathrm{~cm}^{-1}$ with two color ionization without finding any additional peaks. As the second color, we used both 266 and $193 \mathrm{~nm}$ to ensure that we would not miss any excitation due to a high ionization potential.

The keto tautomers are missing in the R2PI spectra in all cases. In unsubstituted guanine, three additional low-energy tautomers exist that each have the $\mathrm{N} 7 \mathrm{H}$ rather than the $\mathrm{N} 9 \mathrm{H}$ form and thus have no equivalent form with the 9-substituted compounds. These are the $\mathrm{N} 7 \mathrm{H}$ enol, at approximately $4.5 \mathrm{kcal} /$ $\mathrm{mol}$, and the two $\mathrm{N} 7 \mathrm{H}$ imino-oxo tautomers at approximately $7 \mathrm{kcal} / \mathrm{mol}$, corresponding to A, B, and C, respectively, in earlier R2PI assignments. ${ }^{7}$ The N9H imino-oxo tautomers of guanine are at considerably higher energies of $15-17 \mathrm{kcal} / \mathrm{mol}$, comparable to the corresponding tautomers in the 9-substituted compounds listed in Table 1. The near-IR-UV data also establish the guanosines to be of the intramolecularly hydrogenbonded cis form, based on the absence of the free $\mathrm{C}^{\prime} 5-\mathrm{O}-\mathrm{H}$ stretch.

IR-UV Double-Resonance Spectra. Figure 4 shows the IR-UV double-resonance spectra of guanosine (a), 2-deoxyguanosine (b), and 9-ethyl guanine (c). For an initial analysis, it is useful to compare spectra of structurally related molecules, while a more complete analysis requires comparison with ab initio computations. By comparing the IR-UV doubleresonance spectra of the guanosines with that of 9-ethyl guanine, we can distinguish ring modes versus sugar modes. By comparing the spectra of guanosine and 2-deoxyguanosine, we may distinguish contributions of hydroxyls in the second and third 
Enol

\section{Keto}

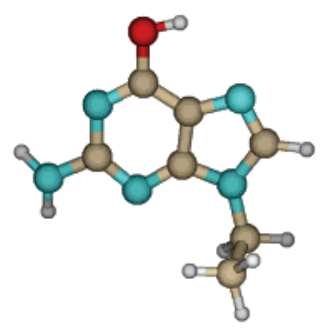

\section{Guanosine}
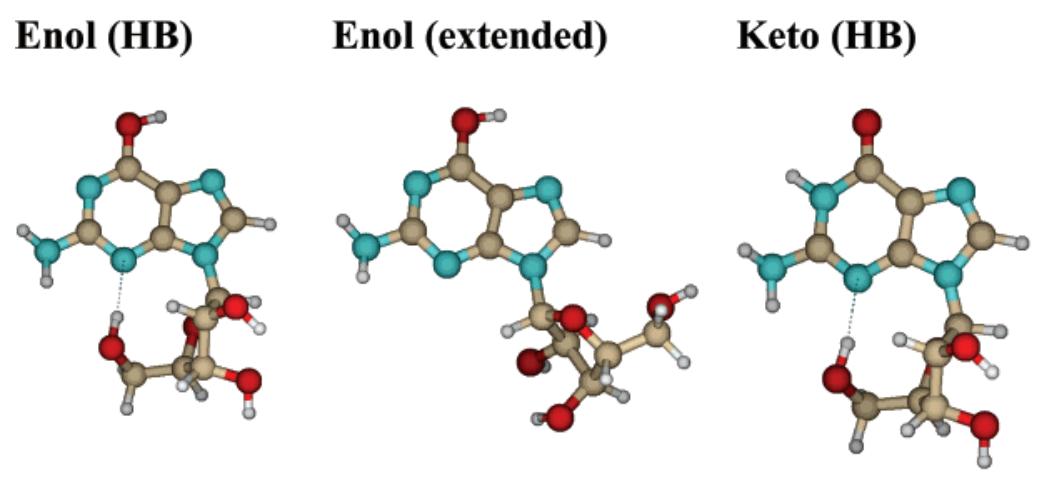

\section{Keto (Extended)}

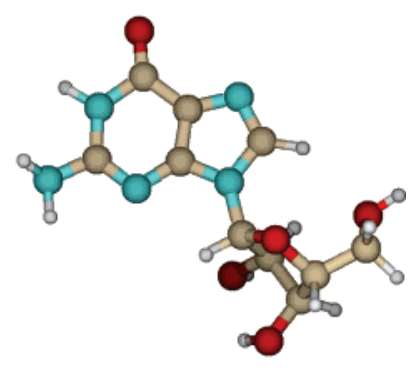

\section{2-Deoxyguanosine}
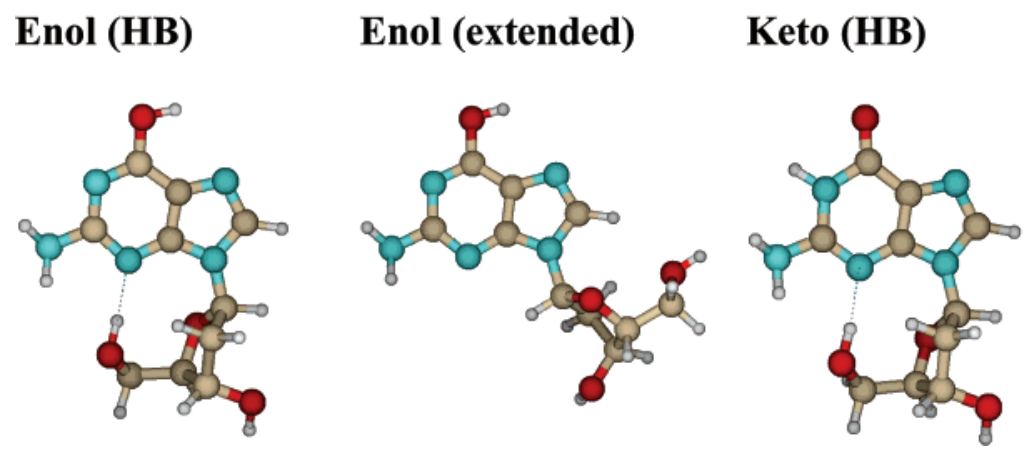

\section{Keto (Extended)}

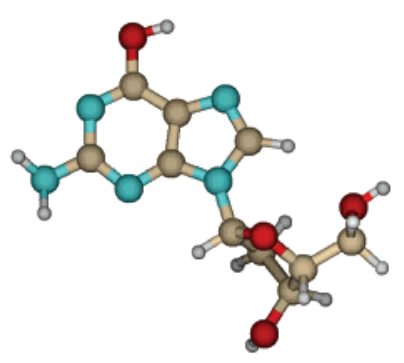

Figure 2. The lowest energy structures of 9-ethyl guanine, guanosine, and 2-deoxyguanosine.

TABLE 1: Relative Stabilities (kcal/mol), Calculated at the RI-MP2 Level, Including Zero-Point Vibrational Energy

\begin{tabular}{|c|c|c|c|c|c|c|}
\hline structure & guanine & $\begin{array}{l}\text { ethyl } \\
\text { guanine }\end{array}$ & $\begin{array}{l}\text { guanosine } \\
\text { extended }\end{array}$ & $\begin{array}{l}\text { guanosine } \\
\text { H-bonded }\end{array}$ & $\begin{array}{l}\text { deoxyguanosine } \\
\text { extended }\end{array}$ & $\begin{array}{c}\text { deoxyguanosine } \\
\text { H-bonded }\end{array}$ \\
\hline keto & 0.00 & 0.00 & 0.00 & -6.91 & 0.00 & -6.83 \\
\hline enol & 1.49 & 1.40 & 1.38 & -6.16 & 1.13 & -5.91 \\
\hline ia & 36.24 & 33.02 & 32.97 & 23.19 & 31.89 & 24.57 \\
\hline $\mathrm{ib}$ & 30.64 & 27.67 & 27.29 & 18.34 & 26.63 & 19.58 \\
\hline n3a & 15.62 & 15.32 & 11.96 & & 15.04 & \\
\hline $\mathrm{n} 3 \mathrm{~b}$ & 17.30 & 16.92 & 13.00 & & 16.45 & \\
\hline oh1a & 32.57 & 31.24 & 30.97 & 22.61 & 30.86 & 23.71 \\
\hline oh1b & 25.74 & 24.54 & 24.06 & 16.12 & 24.11 & 17.09 \\
\hline oh $2 a$ & 39.83 & 38.58 & 38.32 & 30.31 & 38.23 & 31.37 \\
\hline oh $2 b$ & 32.26 & 31.12 & 30.59 & 22.97 & 30.77 & 23.88 \\
\hline
\end{tabular}

position on the sugar ring. The peaks marked in red appear in all three spectra and belong to guanine ring modes that are common to each molecule. The peaks marked in green in trace a are associated with $\mathrm{C}^{\prime} 2-\mathrm{OH}$ modes that are absent in 2-deoxyguanosine, trace b. The remaining peaks are due to the substituent or to combination modes involving the substituent.
Figures 5-7 show the IR-UV double-resonance spectra compared to calculated frequencies of the various structures. Comparison of the calculated frequencies of each structure with the experimental IR spectra shows the enol form to be the best match for each of the three compounds for several reasons. Most notably, the $\mathrm{C}=\mathrm{O}$ stretch mode, which we calculated at about $1745 \mathrm{~cm}^{-1}$ for the keto form, does not appear in the experi- 
TABLE 2: Comparison of Total Energies $\left(E_{\mathrm{h}}\right)$ Calculated with RI-MP2 and RI-DFT-D Methods

\begin{tabular}{cccccc}
\hline & guanine & $\begin{array}{c}\text { ethyl } \\
\text { guanine }\end{array}$ & $\begin{array}{c}\text { guanosine } \\
\text { extended }\end{array}$ & $\begin{array}{c}\text { guanosine } \\
\text { H-bonded }\end{array}$ & $\begin{array}{c}\text { deoxyguanosine } \\
\text { extended }\end{array}$ \\
\hline ktructure (method) & -542.8 & -621.5 & -1039.3 & -1039.3 & -964.0 \\
keto (DFT-D) & -539.4 & -617.5 & -1032.9 & -1032.9 & -964.0
\end{tabular}

mental spectra. Furthermore, those forms would show a single strong peak in the range of $1600-1650 \mathrm{~cm}^{-1}$ due to the $\mathrm{C}-\mathrm{NH}$ stretch for the imino form or a symmetric $\mathrm{NH}_{2}$ bend for the keto form. By contrast, the enol form has three strong modes of roughly equal intensity, within a range of about 1600-1650 $\mathrm{cm}^{-1}$. These are the $\mathrm{OH}$ bending frequencies associated with the $\mathrm{C}-\mathrm{C}$ stretching of the ring, the $\mathrm{C}-\mathrm{C}$ stretching, and the $\mathrm{NH}_{2}$ symmetric bending. Furthermore, we see a strong set of peaks at about $1275 \mathrm{~cm}^{-1}$, which we attribute to a strong inplane $\mathrm{OH}$ bending mode, and at about $1425 \mathrm{~cm}^{-1}$, which we attribute to a ring pinching mode of the guanine. Both of these strong modes are absent in the two keto forms. The only other strong guanine mode would be a NH bending vibration for the imino form at about $1100 \mathrm{~cm}^{-1}$, which we do not observe.

9-Ethyl Guanine. Figure 5 shows the experimental IR-UV double-resonance spectrum (top trace). The stick spectra represent the DFT-D frequencies of the three lowest energy structures (traces $b-d$ ) and the MP2 frequencies for the enol form for comparison (trace e). Several peaks in the 9-ethyl guanine IR-UV double-resonance spectrum are absent in the corresponding spectra for guanosine and 2-deoxyguanosine. We note, in particular, the peaks appearing at 1444, 1363, and 957

(a)

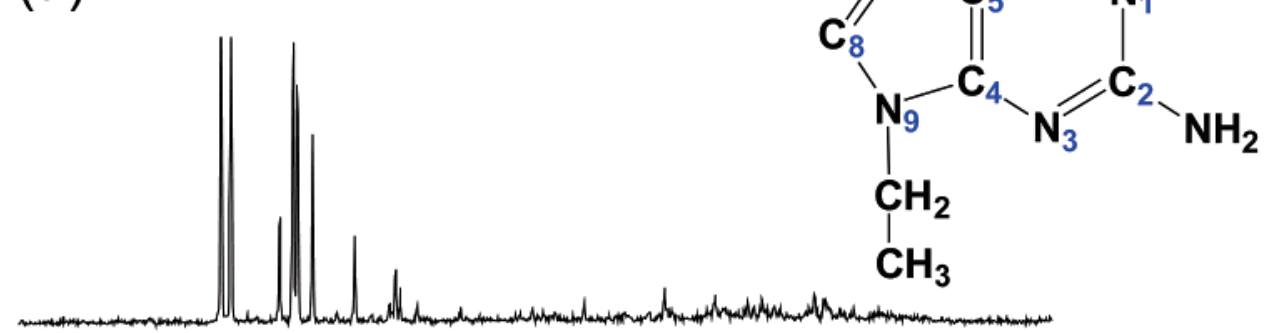

(b)

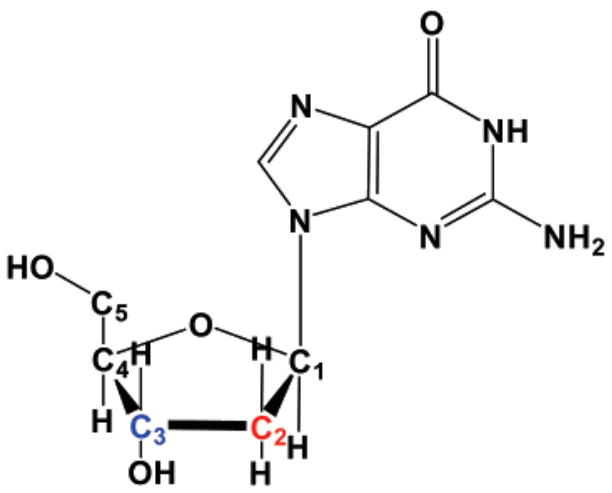

(c)

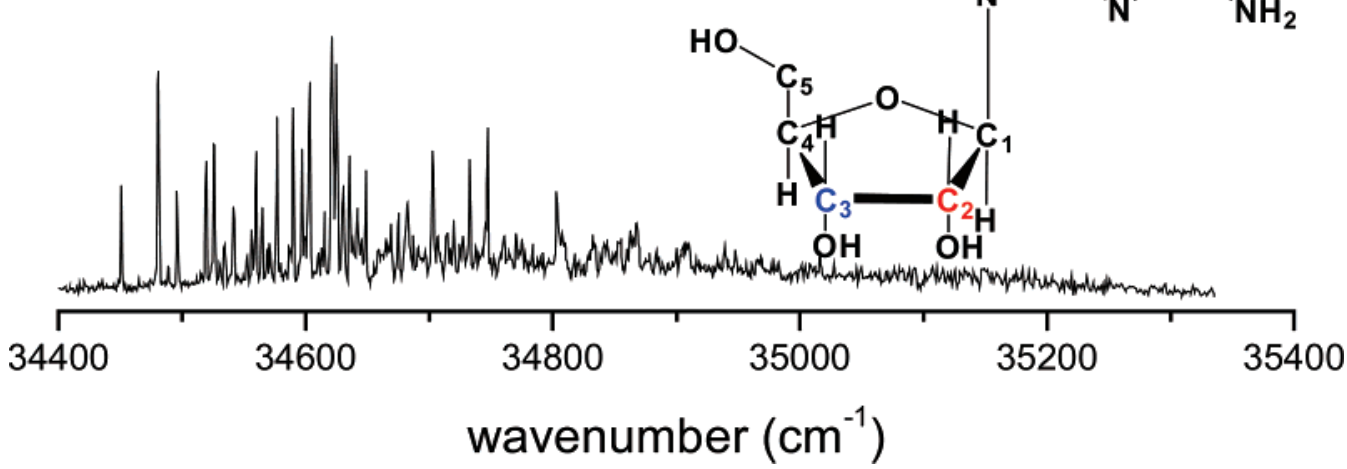

Figure 3. R2PI spectra of 9-ethyl guanine (a), 2-deoxyguanosine (b), and guanosine (c), scanned in the region of $34400-35400 \mathrm{~cm}^{-1}$. On the basis of UV-UV double resonance (not shown) for each molecule, all of the peaks in this region belong to one conformation. 
(a)

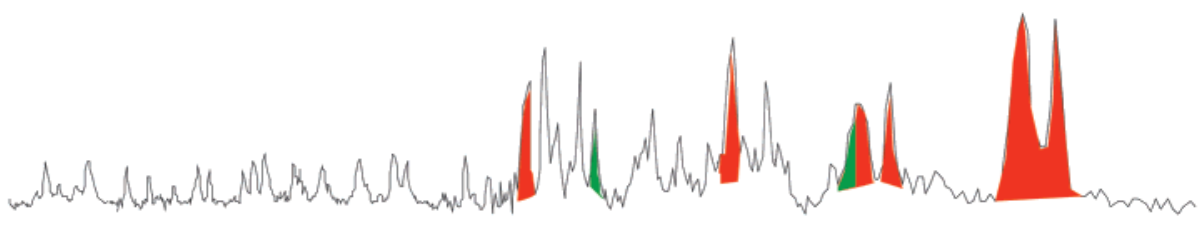

(b)

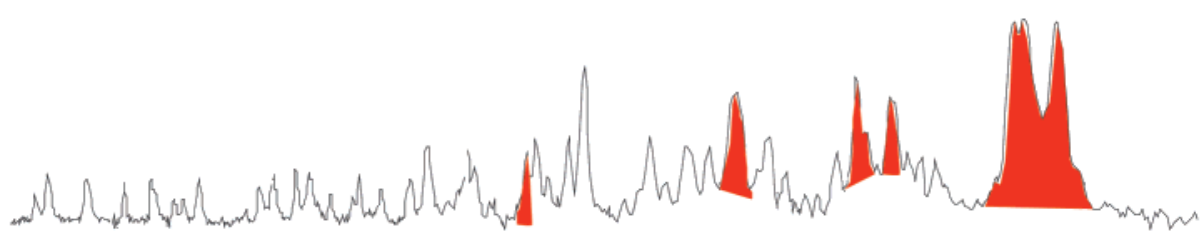

(c)

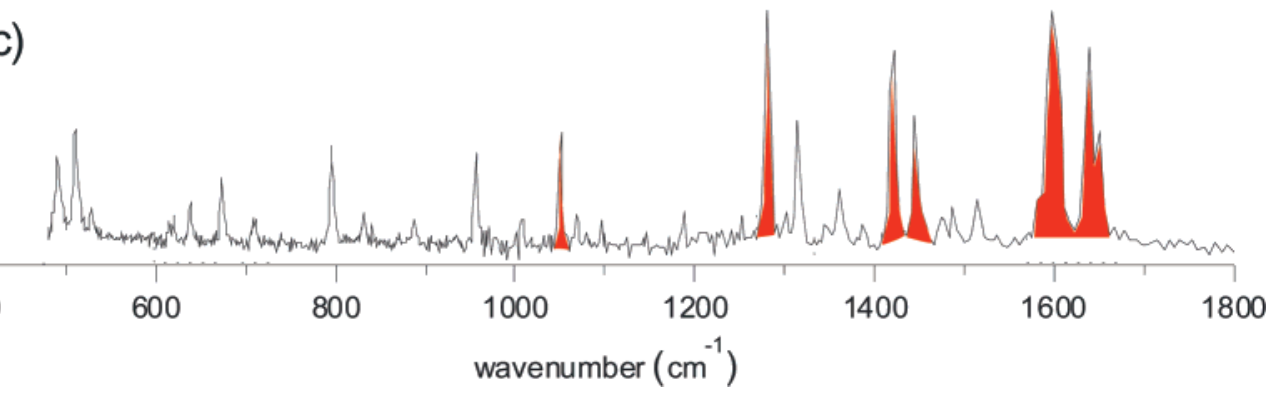

Figure 4. The mid-IR spectra in the region $\left(480-1800 \mathrm{~cm}^{-1}\right.$ ) of guanosine (a), 2-deoxyguanosine (b), and 9-ethyl guanine (c). Red peaks are due to modes of the guanine chromophore; green peaks indicate $\mathrm{C}^{\prime} 2-\mathrm{OH}$ modes.

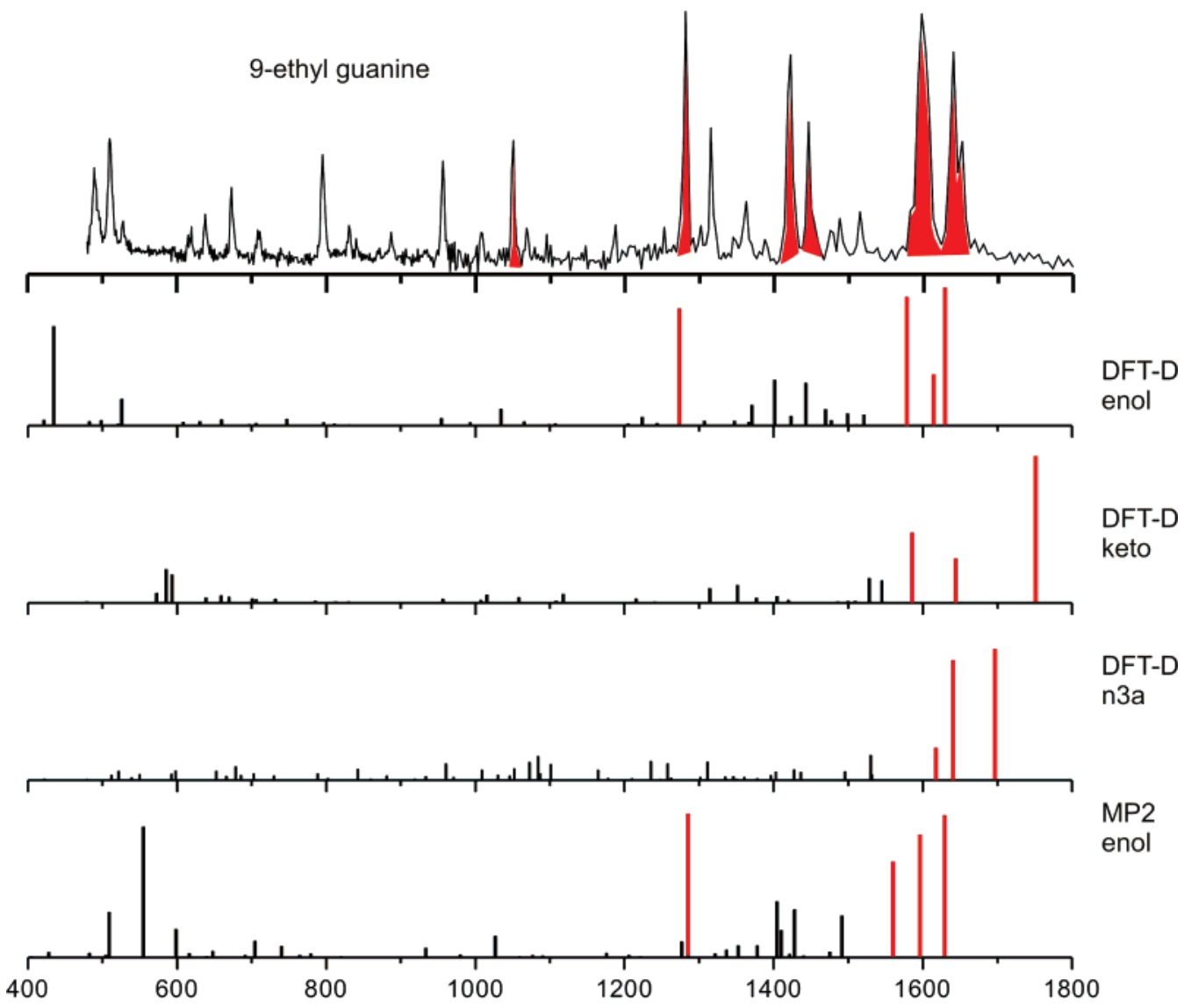

Figure 5. The experimental mid-IR spectrum of 9-ethyl guanine (top panel) and the calculated frequencies of the lowest energy structures.

$\mathrm{cm}^{-1}$. We assign the first peak as the $\mathrm{CH}_{2}$ symmetric bending of the ethyl group, the second peak as the $\mathrm{CH}_{3}$ umbrella stretch, and the third peak as the $\mathrm{C}-\mathrm{C}$ stretching mode of the ethyl. The calculations predict these peaks at 1433, 1339, and 988 $\mathrm{cm}^{-1}$, respectively; however, their intensity is not reproduced properly by the computations at either the MP2 or the DFT level. Overall, the enol form exhibits the best fit, with a better fit for the DFT-D method than that for the MP2 method. 

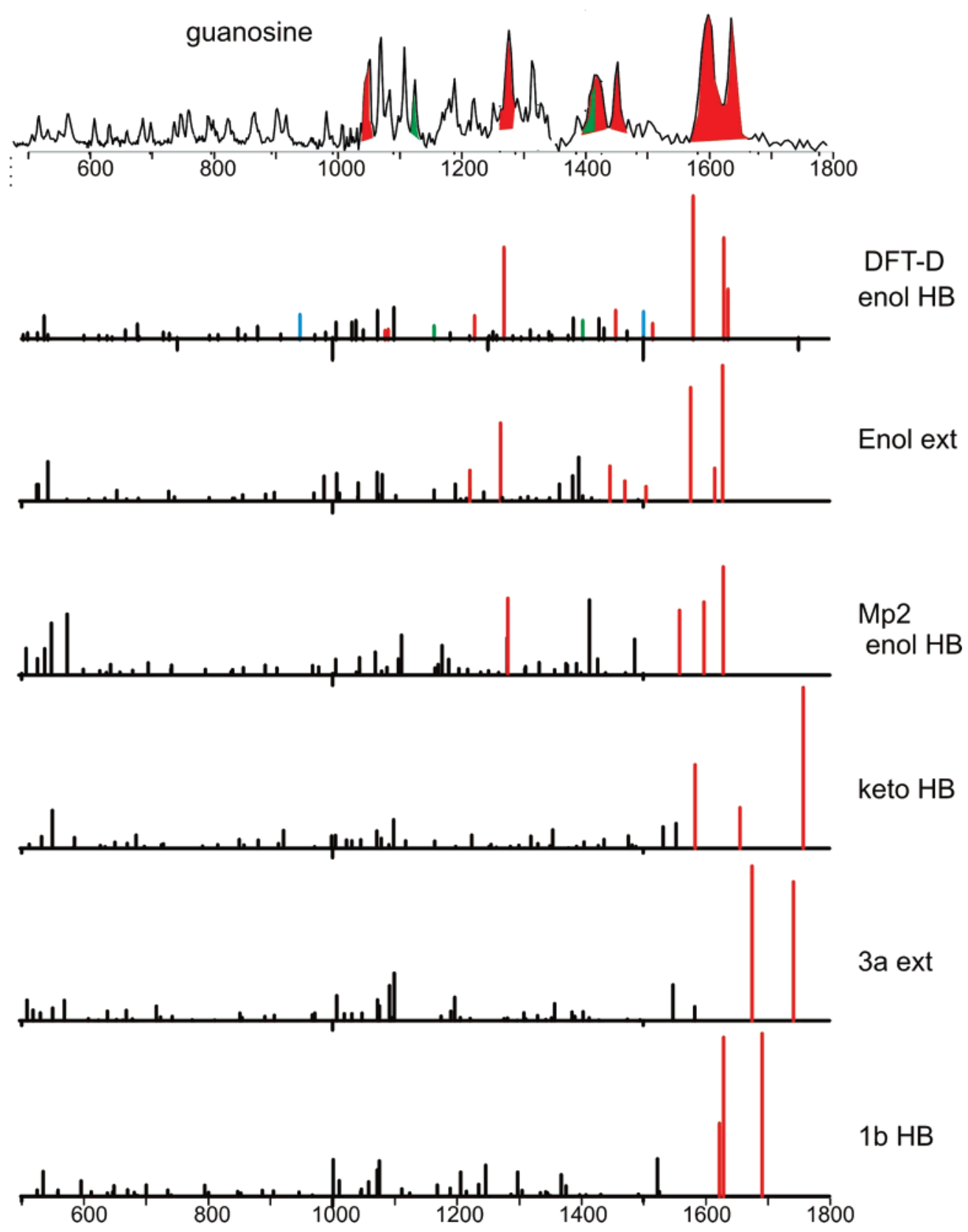

Figure 6. The experimental mid-IR spectrum of guanosine (top panel) and the calculated frequencies of the lowest energy structures.

Guanosine. Figure 6 shows the experimental IR-UV doubleresonance spectrum (top trace). The stick spectra represent the frequencies of the five lowest energy structures, the hydrogenbonded (b) and extended (c) enol forms, the hydrogen-bonded keto (e), extended $3 \mathrm{a}(\mathrm{f})$, and hydrogen-bonded $1 \mathrm{~b}$ form $(\mathrm{g})$. We also show the MP2 results for the hydrogen-bonded enol structure in trace $d$.

Similarly to 9-ethyl guanine, we can exclude all of the keto forms as possible structures, consistent with the near-IR results. The spectra in traces $b$ and $c$ are very similar, with mediumintensity peaks at 948 and at $1501 \mathrm{~cm}^{-1}$ in b, marked in blue, that are absent in $\mathrm{c}$ as the main difference. These peaks belong to modes of the hydrogen-bonded $\mathrm{C}^{\prime} 5-\mathrm{OH}$ of the sugar. Their modest intensity precludes a very confident assignment with experimental data; however, the earlier near-IR data strongly suggest the hydrogen-bonded form. We associate the two peaks marked in green with $\mathrm{C}^{\prime} 2-\mathrm{OH}$ modes. This assignment is consistent with the 2-deoxyguanosine results, presented in Figure 7.
The remaining peaks involve the sugar modes, most of which are much more difficult to assign due to the coupling between different modes and less confident predictions of the calculations in the region below $1000 \mathrm{~cm}^{-1}$.

2-Deoxyguanosine. Figure 7 shows the IR-UV doubleresonance spectrum of 2-deoxyguanosine in trace $a$. Traces $b-d$ show calculated frequencies for the hydrogen-bonded enol form. Panels $\mathrm{b}$ and c compare between guanosine and 2-deoxyguanosine, and panels $b$ and $d$ compare between results from DFT-D and MP2 methods.

The only difference between guanosine and 2-deoxyguanosine is that the $\mathrm{OH}$ in the $\mathrm{C}^{\prime} 2$ position of the sugar is replaced by a hydrogen atom. Therefore, we should expect the disappearance of the peak which we assigned in guanosine to the $\mathrm{C}^{\prime} 2-\mathrm{OH}$ modes. Because of the overlap with the $\mathrm{C}-\mathrm{N}$ stretching mode of the guanine ring, we still see a peak at about $1500 \mathrm{~cm}^{-1}$, which however is much narrower in comparison to that in guanosine. The sugar ring modes in the region of 1041-1134 


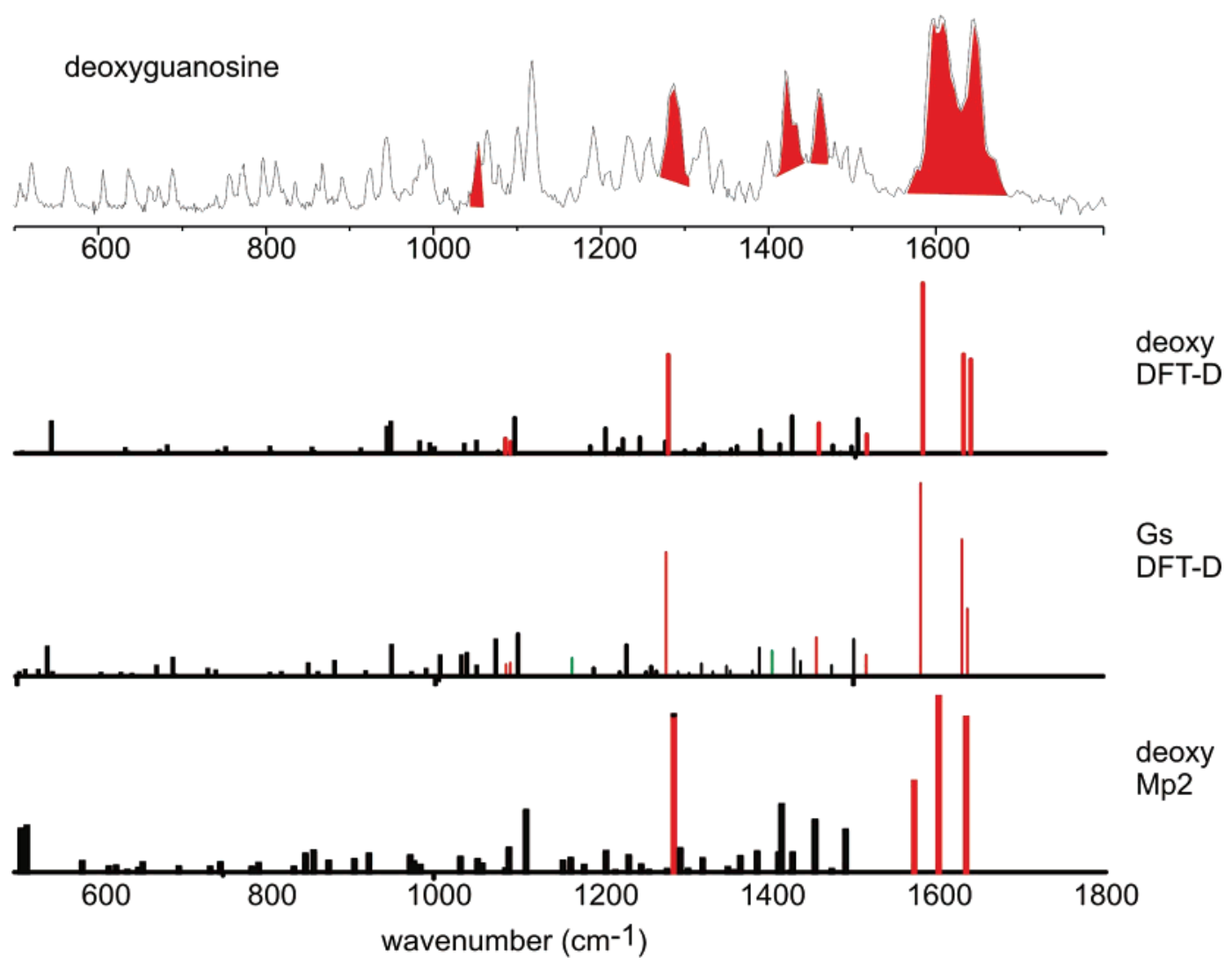

Figure 7. The experimental mid-IR spectrum of 2-deoxyguanosine (top panel) and the calculated frequencies of the lowest energy structures. The guanosine calculation (third trace) is shown for comparison.

$\mathrm{cm}^{-1}$ differ between the two spectra; however, the analysis is hampered by strong coupling.

\section{Summary}

Most of the structural studies of biomolecules in the gas phase so far are determined by spectroscopy in the IR range of 3000$4000 \mathrm{~cm}^{-1}$, where it is possible to measure the $\mathrm{OH}, \mathrm{NH}$, and $\mathrm{NH}_{2}$ symmetric and antisymmetric modes. This region is very sensitive to structural changes. The mid-IR region of 500-2000 $\mathrm{cm}^{-1}$ usually contains a complicated series of absorptions (e.g., bending vibrations) and is more difficult to analyze. Widely tunable mid-IR sources are not readily available, and much less research is done in this region as a result. However, subtle structural information, such as side-chain conformations, is, in principle, encoded in the spectra at these wavelengths. Furthermore, this region is important because it can provide a valuable test of theoretical calculations.

We obtained the IR absorption spectrum of 9-ethyl guanine, guanosine, and 2-deoxyguanosine isolated in the gas phase in the mid-IR region, between 450 and $1800 \mathrm{~cm}^{-1}$. These spectra contain a large number of sharp resonances that can be used for structural assignment by comparison with the theoretical spectra of a set of possible geometries. Calculations show that the keto hydrogen-bonded conformers of guanosine, 2-deoxyguanosine, and the keto form of 9-ethyl guanine are the most stable structure, as is the keto form of unsubstituted guanine. However, it appears that we observe neither the oxo-imino form, which is observed for guanine, nor the keto form, which is absent in guanine also. Helium droplet data, obtained by Roger Miller and co-workers, show that the keto form of guanine can exist in the gas phase. It is therefore plausible that the fact that we do not observe the keto form for any of the guanosines in
R2PI experiments is due to a tautomer-selective short excitedstate lifetime.

The sugar modes are well resolved and add information to the earlier data from the near-IR, but they continue to pose an important challenge for further refinement of theoretical computations. This low-frequency range serves as a stringent test of computational methods. Judging by the most prominent lines, such as those around $1600 \mathrm{~cm}^{-1}$, it appears that the novel DFT-D approach provides promising agreement with experiment, superior to that resulting from the MP2 approach.

Acknowledgment. We gratefully acknowledge the support by the Stichting voor Fundamenteel Onderzoek der Materie (FOM) in providing the required beam time on FELIX and highly appreciate the skillful assistance by the FELIX staff. This material is based upon work supported by the National Science Foundation under Grant No. CHE-0615401. M.K. and P.H. acknowledge the support by Grants Nos. LC512 (from the Ministry of Education of the Czech Republic) and 203/05/009 (from the Grant Agency of the Czech Republic).

\section{References and Notes}

(1) Nir, E.; Grace, L. I.; Brauer, B.; de Vries, M. S. REMPI Spectroscopy of Jet Cooled Guanine. J. Am. Chem. Soc. 1999, 121, 48964897.

(2) Nir, E.; Imhof, P.; Kleinermanns, K.; de Vries, M. S. REMPI Spectroscopy of Laser Desorbed Guanosines. J. Am. Chem. Soc. 2000, 122, 8091-8092.

(3) Piuzzi, F.; Mons, M.; Dimicoli, I.; Tardivel, B.; Zhao, Q. Ultraviolet Spectroscopy and Tautomerism of the DNA Base Guanine and its Hydrate Formed in a Supersonic Jet. Chem. Phys. 2001, 270, 205-14.

(4) Mons, M.; Dimicoli, I.; Piuzzi, F.; Tardivel, B.; Elhanine, M. Tautomerism of the DNA Base Guanine and its Methylated Derivatives as Studied by Gas-Phase Infrared and Ultraviolet Spectroscopy. J. Phys. Chem. A 2002, 106, 5088-5094. 
(5) Chin, W.; Mons, M.; Dimicoli, I.; Piuzzi, F.; Tardivel, B.; Elhanine, M. Tautomer Contribution's to the Near UV Spectrum of Guanine: Towards a Refined Picture for the Spectroscopy of Purine Molecules. Eur. Phys. J. D 2002, 20, 347-355.

(6) Chin, W.; Mons, M.; Piuzzi, F.; Tardivel, B.; Dimicoli, I.; Gorb, L.; Leszczynski, J. Gas Phase Rotamers of the Nucleobase 9-Methyl Guanine Enol and Its Monohydrate: Optical Spectroscopy and Quantum Mechanical Calculations. J. Phys. Chem. A 2004, 108, 8237-8243.

(7) Mons, M.; Piuzzi, F.; Dimicoli, I.; Gorb, L.; Leszczynski, J. NearUV Resonant Two-Photon Ionization Spectroscopy of Gas Phase Guanine: Evidence for the Observation of Three Rare Tautomers. J. Phys. Chem. A 2006, 110, 10921-10924.

(8) Choi, M. Y.; Miller, R. E. Four Tautomers of Isolated Guanine from Infrared Laser Spectroscopy in Helium Nanodroplets. J. Am. Chem. Soc. 2006, 128, 7320-7328.

(9) Kleinermanns, K. 2006, private communication.

(10) Choi, M. Y.; Douberly, G. E.; Falconer, T. M.; Lewis, W. K.; Lindsay, C. M.; Merritt, J. M.; Stiles, P. L.; Miller, R. E. Infrared Spectroscopy of Helium Nanodroplets: Novel Methods for Physics and Chemistry. Int. Rev. Phys. Chem. 2006, 25, 15-75.

(11) Broo, A.; Holmen, A. Ab Initio MP2 and DFT Calculations of Geometry and Solution Tautomerism of Purine and Some Purine Derivatives. Chem. Phys. 1996, 211, 147-161.

(12) Sobolewski, A. L.; Domcke, W. On the Mechanism of Nonradiative Decay of DNA Bases: Ab Initio and TDDFT Results for the Excited States of 9H-Adenine. Eur. Phys. J. D 2002, 20, 369-374.

(13) Schultz, T.; Samoylova, E.; Radloff, W.; Hertel, I. V.; Sobolewski, A. L.; Domcke, W. Efficient Deactivation of a Model Base Pair via ExcitedState Hydrogen Transfer. Science 2004, 306, 1765-1768.

(14) Sobolewski, A. L.; Domcke, W. Relevance of Electron-Driven Proton-Transfer Processes for the Photostability of Proteins. ChemPhysChem 2006, 7, 561-564.

(15) Kang, H.; Jung, B.; Kim, S. K. Mechanism for Ultrafast Internal Conversion of Adenine. J. Chem. Phys. 2003, 118, 6717-6719.

(16) Canuel, C.; Mons, M.; Piuzzi, F.; Tardivel, B.; Dimicoli, I.; Elhanine, M. Excited States Dynamics of DNA and RNA Bases: Charac- terization of a Stepwise Deactivation Pathway in the Gas Phase. J. Chem. Phys. 2005, 122, 074316/1-074316/6.

(17) Marian, C. M. A New Pathway for the Rapid Decay of Electronically Excited Adenine. J. Chem. Phys. 2005, 122, 104314/1-104314/13.

(18) Nir, E.; Kleinermanns, K.; Grace, L.; de Vries, M. S. On the Photochemistry of Purine Nucleobases. J. Phys. Chem. A 2001, 105, 51065110 .

(19) Nir, E.; Hunig, I.; Kleinermanns, K.; de Vries, M. S. Conformers of Guanosines and their Vibrations in the Electronic Ground and Excited States, as Revealed by Double-Resonance Spectroscopy and Ab Initio Calculations. ChemPhysChem 2004, 5, 131-137.

(20) Bakker, J. M.; Aleese, L. M.; von Helden, G.; Meijer, G. Fingerprint IR Spectroscopy to Probe Amino Acid Conformations in the Gas Phase. Phys. Rev. Lett. 2003, 91, 203003/2-203003/4.

(21) Bakker, J. M.; Satink, R. G.; von Helden, G.; Meijer, G. Infrared Photodissociation Spectroscopy of Benzene-Ne,Ar Complex Cations. Phys. Chem. Chem. Phys. 2002, 4, 24-33.

(22) Meijer, G.; de Vries, M. S.; Hunziker, H. E.; Wendt, H. R. Laser Desorption Jet-Cooling of Organic-Molecules - Cooling Characteristics and Detection Sensitivity. Appl. Phys. B 1990, 51, 395-403.

(23) Oepts, D.; van der Meer, A. F. G.; van Amersfoort, P. W. The Free-Electron-Laser User Facility Felix. Infrared Phys. Technol. 1995, 36, 297-308.

(24) Jurečka, P.; Černy, J.; Hobza, P.; Salahub, D. R. Density Functional Theory Augmented with an Empirical Dispersion Term. Interaction Energies and Geometries of 80 Noncovalent Complexes Compared with Ab Initio Quantum Mechanics Calculations. J. Comput. Chem. 2007, 28, 555-569.

(25) Jurečka, P.; Černý, J.; Hobza, P.; Valdés, H. J. Phys. Chem. A 2007, $111,1146-1154$.

(26) Tao, J. M.; Perdew, J. P.; Staroverov, V. N.; Scuseria, G. E. Climbing the Density Functional Ladder: Nonempirical Meta-Generalized Gradient Approximation Designed for Molecules and Solids. Phys. Rev. Lett. 2003, 91, 146401/1-146401/4.

(27) Ahlrichs, R.; Bär, M.; Häser, M.; Horn, H.; Kölmel, C. ElectronicStructure Calculations on Workstation Computers - the Program System Turbomole. Chem. Phys. Lett. 1989, 162, 165-169. 